\title{
Should Perfusion Scintigraphy Be Performed to Follow Patients with Acute Pulmonary Embolism? If So, When?
}

\author{
Letizia Marconi ${ }^{1}$, Antonio Palla ${ }^{1}$, Lucia Cestelli ${ }^{1}$, Marco Lazzeretti ${ }^{1}$, Laura Carrozzi $^{1}$, Massimo Pistolesi ${ }^{2}$, \\ and Henry Dirk Sostman ${ }^{3-6}$ \\ ${ }^{I}$ Respiratory Unit, Department of Surgical, Medical and Molecular Pathology, and Critical Care, University of Pisa, Pisa, Italy; \\ ${ }^{2}$ Section of Respiratory Medicine, Department of Experimental and Clinical Medicine, University of Florence, Florence, Italy; \\ ${ }^{3}$ Houston Methodist Hospital, Houston, Texas; ${ }^{4}$ Weill Cornell Medicine, New York, New York; ${ }^{5}$ Methodist Research Institute, Houston, \\ Texas; and ${ }^{6}$ Houston Methodist Hospital System, Houston, Texas
}

This investigation evaluated the changes of pulmonary perfusion at 4 different points of follow-up within $1 \mathrm{y}$ in patients with pulmonary embolism (PE) and the factors predictive of complete or incomplete recovery of pulmonary perfusion. Methods: Patients with symptomatic $P E$ underwent perfusion lung scintigraphy and blood gas analysis within $48 \mathrm{~h}$ from clinical presentation, after $1 \mathrm{wk}$, and after 1, 6, and $12 \mathrm{mo}$; echocardiography was performed at baseline and after 6 and 12 mo. All perfusion lung scintigraphy scans were examined by 2 expert nuclear medicine physicians with a scoring method that attributed a score of $0,0.5$, or 1 for extension (maximum score, 18) to the presence of perfusion defects (PD), both at baseline and on each follow-up scan. Results: Among 183 patients who completed $1 \mathrm{y}$ of follow-up, the median baseline PD score was 8.2; it decreased significantly at each follow-up time point until 6 mo $(P<$ $0.001)$. Median baseline alveolar-arterial difference in oxygen partial pressure $\left(\mathrm{PA}-\mathrm{aO}_{2}\right)$ was 50.9 and decreased significantly up to $1 \mathrm{mo}$ $(P<0.001)$; median pulmonary artery systolic pressure (PAsP) was $45.9 \mathrm{~mm} \mathrm{Hg}$ and decreased significantly until $12 \mathrm{mo}(P<0.001)$. A correlation was found between $\mathrm{PD}$ and both $\mathrm{PA}-\mathrm{aO}_{2}(P<0.05)$ and PAsP $(P<0.05)$. We found a correlation between $\mathrm{PD} \neq 0$ and $\mathrm{PAsP} \geq 40 \mathrm{~mm} \mathrm{Hg}$ at $12 \mathrm{mo}(P<0.05)$; in $6(3.3 \%)$ of these patients such a correlation was still present after 24 mo, suggesting they could develop chronic thromboembolic pulmonary hypertension. Low baseline PD (odds ratio, 0.80; $P<0.0001$ ) and high 1 -wk percent recovery (odds ratio, 1.04; $P<0.0001$ ) were predictive factors of complete 6-mo recovery. Conclusion: Perfusion scintigraphy may be useful to follow patients with $\mathrm{PE}$. The follow-up should consist of 3 steps: the baseline examination, which reflects the severity of PE; the scan at $1 \mathrm{wk}$, which indicates the early amount of reperfusion; and the scan at 6 mo, which demonstrates the maximum attainable recovery. Patients with incomplete recovery and persistence of pulmonary hypertension on the 24-mo control should be further studied for possible development of chronic thromboembolic pulmonary hypertension.

Key Words: pulmonary embolism; pulmonary lung scintigraphy; follow-up

J Nucl Med 2019; 60:1134-1139

DOI: 10.2967/jnumed.118.222737

Received Oct. 30, 2018; revision accepted Dec. 18, 2018.

For correspondence or reprints contact: Letizia Marconi, Unità di Pneumologia, Università e Ospedale di Pisa, Via Paradisa 2, Pisa, Italy.

E-mail: letizia.marconi@med.unipi.it

Published online May 23, 2019.

COPYRIGHT (C 2019 by the Society of Nuclear Medicine and Molecular Imaging.
$\mathbf{T}$ he risk of adverse events in patients with acute pulmonary embolism (PE) continues beyond the acute phase, since PE may recur both in the early days after the acute event and after months or years (1-3) and may, in a few cases, result in pulmonary hypertension $(4,5)$. Patients at high risk for unfavorable outcomes, who may benefit from prolonged anticoagulation, should therefore be identified early. However, currently there is no practical way to know whether a patient is at high risk of early and late complications. Recently, Pesavento et al. demonstrated that the persistence of a residual pulmonary arterial obstruction on perfusion lung scintigraphy (PLS) 6 mo after an index event represents a risk factor for recurrence and for the development of chronic postembolic pulmonary hypertension after a first episode of PE (6). This suggests a role for scintigraphic follow-up to identify patients at higher risk for adverse events after PE. However, this result needs out-of-sample confirmation. Even if confirmed, it would still not be clear when the scintigraphic follow-up would be most effective. Is a single 6mo follow-up of the incident event the most appropriate metric? How does pulmonary perfusion change before and after $6 \mathrm{mo}$ ? Could the adverse outcomes be predicted sooner? Are there correlations between perfusion improvement and functional parameters, such as gas exchange and pulmonary artery pressure, that could help in identifying patients at risk for chronic thromboembolism? Are there factors that facilitate or hamper restoration of pulmonary perfusion?

The main purpose of the present study was to investigate, in patients with a symptomatic episode of acute PE, the changes in pulmonary perfusion at 4 different times of follow-up within $1 \mathrm{y}$. Secondary purposes were to investigate, first, the presence of factors predictive of complete or incomplete recovery of pulmonary perfusion at different follow-up times and, second, the correlations between changes in pulmonary perfusion and both pulmonary gasexchange and pulmonary artery pressure that might be useful to identify patients at risk for late complications.

\section{MATERIALS AND METHODS}

This study was conducted in the Respiratory Unit of the University Hospital of Pisa, Italy; all patients had been diagnosed with a symptomatic episode of PE by CT angiography (CTA) or by PLS combined with a high clinical probability of $\mathrm{PE}$, according to previously published criteria $(7,8)$.

According to Respiratory Unit policy, all patients were routinely scheduled for follow-up PLS controls at $1 \mathrm{wk}, 1 \mathrm{mo}, 6 \mathrm{mo}$, and $1 \mathrm{y}$ after the incident event $(9,10)$. If PE was diagnosed initially by CTA, a 
baseline PLS was also obtained according to Respiratory Unit policy within $24-48 \mathrm{~h}$ of the CTA. The PLS scans were reviewed using a single-center, longitudinal, observational design. The institutional review board approved this study, and the requirement to obtain informed consent was waived.

The following baseline data were collected: age, sex, and presence of cardiovascular disease, chronic obstructive pulmonary disease, active cancer, previous venous thromboembolism, or current symptomatic deep vein thrombosis. Patients were classified as having transiently provoked $\mathrm{PE}$ if they reported any of the following risk factors: recent surgery, immobilization, fracture, or estrogen therapy. All patients without these risk factors were regarded as having unprovoked PE. Blood gas analysis was performed in association with the scans while the patients were breathing room air, except for a limited number

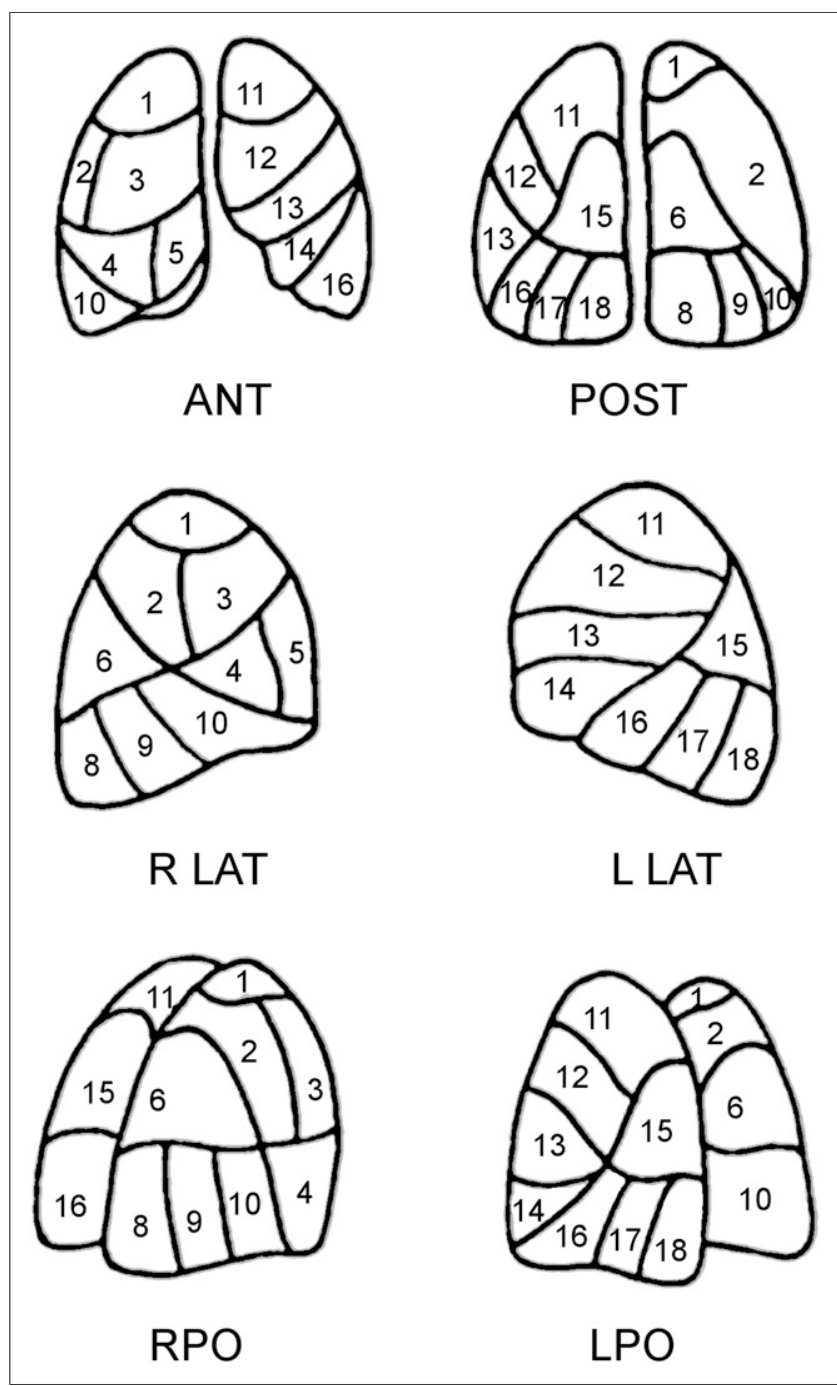

FIGURE 1. Reading scheme reporting anatomic distribution of lung segments. Right lung (1-10): upper lobe (apical [1]; posterior [2]; anterior [3]); middle lobe (lateral [4]; medial [5]); lower lobe (superior [6]; medialbasal $[7 ; 7$ is not shown in the figure because the anterior medial segment could only be visualized on the oblique anterior projection, which is not used in this article]; posterior-basal [8]; lateral-basal [9]; anteriorbasal [10]). Left lung (11-18): upper lobe (apical-posterior [11]; anterior [12]); lingula (superior [13]; inferior [14]); lower lobe (superior [15]; medialbasal [16]; lateral-basal [17]; posterior-basal [18]). ANT = anterior; POST $=$ posterior; $\mathrm{R}$ LAT $=$ right lateral; $\mathrm{L}$ LAT = left lateral; RPO = right posterior oblique; $\mathrm{LPO}=$ left posterior oblique.
TABLE 1

Baseline Characteristics of Patients

\begin{tabular}{|lc|}
\hline \multicolumn{1}{c}{ Characteristic } & Data \\
\hline Mean age \pm SD $(\mathrm{y})$ & $68.56 \pm 13.82$ \\
\hline Male & $79(43.2)$ \\
\hline Deep-vein thrombosis & $69(37.7)$ \\
\hline Previous venous thromboembolism & $15(8.2)$ \\
\hline Cardiovascular disease & $104(56.8)$ \\
\hline Chronic obstructive pulmonary disease & $29(15.8)$ \\
\hline Cancer-associated PE & $33(18.0)$ \\
\hline Transient provoked PE & $48(26.2)$ \\
\hline Unprovoked PE & $102(55.7)$ \\
\hline
\end{tabular}

Data are $n$ followed by percentage in parentheses, except for age.

of patients ( $n=11,5.9 \%)$ who had supplemental oxygen administered. From blood gas analysis, the alveolar-arterial difference in oxygen partial pressure $\mathrm{PA}-\mathrm{aO}_{2}=\left[(760-47) \times \mathrm{FiO}_{2}\right.$ (fraction of inspired oxygen) $]-\left(\mathrm{PaCO}_{2} / 0.8\right)-\mathrm{PaO}_{2}$ (partial pressure arterial oxygen) was calculated. Values of pulmonary artery systolic pressure (PAsP) were calculated by transthoracic echocardiography at baseline and 6 and 12 mo later; if PAsP was higher than $40 \mathrm{~mm} \mathrm{Hg}$ at $1 \mathrm{y}$, a control echocardiogram was performed $2 \mathrm{y}$ after the acute episode.

\section{Lung Perfusion Analysis}

All scans were examined by 2 nuclear medicine specialists who had many years of clinical experience in reading PLS and had participated on the reading committees of several studies of the image database of the Prospective Investigation of Pulmonary Embolism Diagnosis (PIOPED) and the Prospective Investigative Study of Acute Pulmonary Embolism Diagnosis (PISA-PED). Each PLS examination consisted of 6 views (anterior, posterior, right and left lateral, and right and left posterior oblique). The PISA-PED method adopted here was the same successfully used to reread the PIOPED cases, and it is approved by the Society of Nuclear Medicine and Molecular Imaging guidelines $(11,12)$.

To quantify the perfusion impairment at diagnosis and to evaluate changes during follow-up, we used a scoring system originally developed

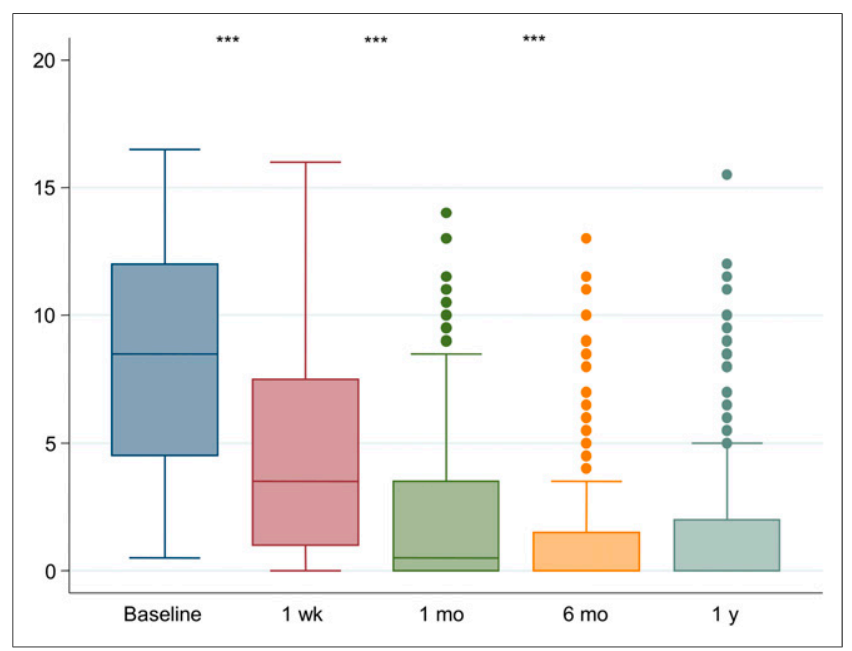

FIGURE 2. Values of PD score at different follow-up times. ${ }^{\star \star \star} P<0.001$. 


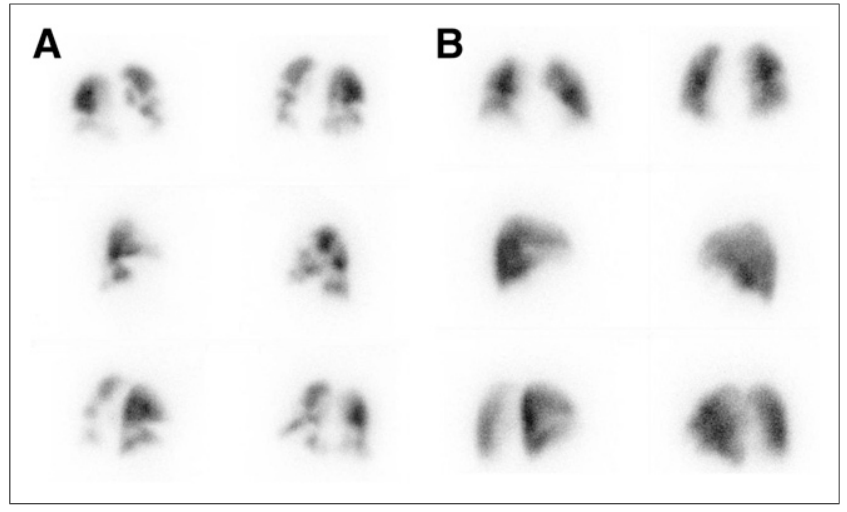

FIGURE 3. Changes in PD and hyperperfusion in 6 different views: anterior and posterior (top), right and left lateral (middle), and right and left posterior oblique (bottom). (A) Baseline PLS at acute PE shows several segmental PDs and shift of perfusion from posterior-inferior to anterior-superior lung regions (hyperperfusion) (PD score $=5.5$, hyperperfusion in upper and middle lobes). (B) PLS 6 mo later shows marked improvement ( $P D$ score $=1.0$, hyperperfusion in upper and middle lobes).

in our institution. The system was based on lung segmental divisions (right lung, 10 segments; left lung, 8 segments). The readers evaluated by consensus the perfusion characteristics of each of the 18 segments reported in the scheme (Fig. 1). In detail, a score of 0 was attributed to a normally perfused segment, 0.5 to a segment with up to a $50 \%$ reduction of perfusion, and 1 to a segment with underperfusion ranging from $50 \%$ to $100 \%$. A total score that could range from 0 to 18 for the 2 lungs was then calculated. The score was assigned at baseline and at each follow-up scan. On the basis of previous clinical observations $(13,14)$, a second parameter was investigated: the presence of areas of hyperperfusion in the upper and middle lung lobes. This is a newly introduced parameter that indicates the appearance of areas of increased perfusion in lung zones normally less perfused: those in the upper and anterior regions. Such a phenomenon is due to redistribution of perfusion to lung regions free of embolization. The presence of hyperperfusion was investigated at baseline and on the follow-up scans.

\section{Statistical Analysis}

Continuous variables were expressed as mean \pm SD. Categoric variables were expressed as numbers and percentages. Changes in perfusion defects (PDs), blood gases, and PAsP over time were graphed as box plots. Correlations were evaluated by the $t$ test of the Pearson correlation. Differences between groups, classified by complete versus incomplete recovery, were evaluated by the Student $t$ test for continuous variables and the $\chi^{2}$ test for categoric variables. The significance level was set at 5\% $(P<$ 0.05 ). Factors conditioning perfusion improvement were investigated by a logistic model using the backward-stepwise approach to select variables.

\section{RESULTS}

The total number of consecutive patients diagnosed with PE during 3 y was 321 . Of those, $80(24.9 \%)$ were excluded because their baseline scintigraphy was performed more than $48 \mathrm{~h}$ after the PE diagnosis; 58 (18.1\%) were lost to follow-up, either for logistic reasons or because they did not return after the baseline scintigraphy. The final study population, therefore, consisted of $183(57 \%)$ patients who had completed the full 1-y follow-up. The baseline characteristics of patients are reported in Table 1.

\section{Recovery of Pulmonary Perfusion}

Changes in PD score at different points of follow-up are reported in Figure 2. The median baseline PD score was 8.2; it significantly decreased on each follow-up step until $6 \mathrm{mo}$, when it became stable. Perfusion recovery was complete in $12 \%$ of patients at 1 wk, in $46 \%$ at $1 \mathrm{mo}$, and in $59 \%$ at both 6 and $12 \mathrm{mo}$.

Hyperperfusion was present in $114(62.3 \%)$ patients at baseline and in $86(46.9 \%), 40(21.9 \%), 21(11.5 \%)$, and 18 (9.8\%) patients at $1 \mathrm{wk}, 1 \mathrm{mo}, 6 \mathrm{mo}$, and $1 \mathrm{y}$, respectively. Figure 3 shows the changes in PD and hyperperfusion from acute embolization (Fig. 3A) to stable conditions (Fig. 3B) after 6 mo.

Median $\mathrm{PA}-\mathrm{aO}_{2}$ at baseline was 50.9; it decreased significantly during the followup until 6 mo (Fig. 4A). Median PAsP at baseline was $45.9 \mathrm{~mm} \mathrm{Hg}$; PAsP values significantly decreased at every follow-up step until 1 y (Fig. 4B).

The correlation of PD with $\mathrm{PA}-\mathrm{aO}_{2}$ and $\mathrm{PAsP}$ at baseline and during follow-up is reported in Table 2. A negative correlation exists between $\mathrm{PD}$ and $\mathrm{PA}-\mathrm{aO}_{2}$ during the entire follow-up $(P<0.05)$. A correlation between $\mathrm{PD}$ and PAsP was found only at 6 mo and $1 \mathrm{y}$ $(P<0.05)$. In particular, a correlation was found $(P<0.05)$ in a subgroup of $10(5 \%)$ patients who still had a pulmonary pressure over $40 \mathrm{~mm} \mathrm{Hg}$ and a residual arterial pulmonary obstruction at 12 mo. In $6(3.3 \%)$ of these patients, such a correlation was still present after 24 mo. The presence of hyperperfusion at the 6-mo control correlated $(P<$ $0.05)$ with a PAsP of at least $40 \mathrm{~mm} \mathrm{Hg}$.

\section{Factors Affecting Perfusion Improvement}

Variables measured at baseline and $1 \mathrm{wk}$ later that were associated with complete recovery were investigated by both univariate

FIGURE 4. Values of $P A-a_{2}(A)$ and PAsP (B) at different follow-up times. ${ }^{\star \star} P<0.01$ ${ }^{* \star \star} P<0.001$. 
TABLE 2

Correlation of PD Versus $\mathrm{PA}-\mathrm{aO}_{2}$ and PAsP at Different Follow-up Times

\begin{tabular}{cccccc}
\hline Correlation & Baseline & 1 wk & 1 mo & 6 mo & $1 \mathrm{y}$ \\
\hline PD vs. PA-aO & $0.29(P<0.001)$ & $0.19(P<0.05)$ & $0.24(P<0.05)$ & $0.20(P<0.05)$ & $0.34(P<0.0001)$ \\
PD vs. PAsP & $0.04(P=0.68)$ & - & - & $0.36(P<0.001)$ & $0.25(P<0.05)$ \\
\hline
\end{tabular}

and multivariate analysis. Among the former, younger age, low PD at baseline, high 1-wk percent recovery, and presence of transiently provoked PE were associated with complete recovery at the 1-mo follow-up, whereas only the first three were related to a complete recovery at 6 mo (Table 3). Low PD at baseline, high 1-wk percent recovery, and presence of transiently provoked PE were associated with complete recovery at 1 mo. Low PD at baseline and high 1-wk percent recovery were associated with complete recovery at 6 mo (Table 4 ). Age showed a borderline significance (Table 4).

\section{DISCUSSION}

After an episode of acute PE, patients should be followed to evaluate the progress of improvement and the potential occurrence of adverse events, both during and after cessation of therapy. To this end, clinical $(1,15,16)$, laboratory (17-19), radiologic (20-23), and echocardiographic $(24,25)$ patterns have been used. Recently, the use of PLS has demonstrated (6) that incomplete restoration of pulmonary blood flow is predictive of recurrence and of arterial pulmonary hypertension. However, in that study, the pulmonary scintigraphy was performed only 6 mo after the acute event, with no information available about earlier (1 wk, $1 \mathrm{mo})$ and later (1 y) times after embolization.

The present study demonstrates that the restoration of pulmonary blood flow is progressive with time until 6 mo after the incident event and that no further improvement is to be expected after that time. Complete recovery of pulmonary blood flow occurred in only $13 \%$ of patients (17\% of those who ultimately recovered) after $1 \mathrm{wk}$ of therapy, in about $50 \%$ of patients $(84 \%$ of those who ultimately recovered) after $1 \mathrm{mo}$, and in $60 \%$ of patients after 6 and 12 mo. Therefore, the control at 6 mo appears mandatory because at this time point patients can be expected to have achieved stable restoration of pulmonary blood flow. At earlier time points, not all patients who will achieve complete resolution of perfusion would be identified, whereas the $1-y$ time point involves a longer delay and provides no additional information. The choice by Pesavento et al. (6) of a 6-mo step of follow-up to investigate the presence of residual obstruction in order to predict late adverse-event results was prescient.

Nevertheless, other follow-up evaluations can be clinically useful. Of course, the severity of PE at baseline allows one to predict a complete perfusion recovery at 6 mo: the less severe the initial reduction of perfusion, the higher will be the probability that a patient will have normal perfusion after $6 \mathrm{mo}$. This is consistent with the data reported by Wartski and Collignon (26), who, in performing PLS at baseline, at $8 \mathrm{~d}$, and after $3 \mathrm{mo}$, found that the greater the initial perfusion deficit, the lower the perfusion recovery that could be expected at follow-up. The extent of perfusion improvement $7 \mathrm{~d}$ after acute $\mathrm{PE}$ is an independent predictor of complete recovery after 6 mo. This means that the earlier that pulmonary perfusion improvement occurs, the higher is the probability that the patient will reach a complete 6-mo resolution. On the basis of the odds ratio, we compute that each 1-point score of

TABLE 3

Variables Associated with Recovery at 1 and 6 Months of Follow-up

\begin{tabular}{|c|c|c|c|c|c|c|}
\hline \multirow[b]{2}{*}{ Variable } & \multicolumn{3}{|c|}{ Recovery at $1 \mathrm{mo}$} & \multicolumn{3}{|c|}{ Recovery at 6 mo } \\
\hline & Incomplete & Complete & $P$ & Incomplete & Complete & $P$ \\
\hline Male & $45(46.9)$ & $30(37.0)$ & 0.187 & $32(50.8)$ & $38(40.9)$ & 0.221 \\
\hline Age $(y)$ & $70.7 \pm 13.6$ & $65.6 \pm 13.9$ & $<0.05$ & $73.2 \pm 11.2$ & $65.5 \pm 14.2$ & $<0.001$ \\
\hline Cardiovascular disease & $54(56.3)$ & $46(56.8)$ & 0.942 & $41(65.1)$ & $54(58.1)$ & 0.378 \\
\hline Chronic obstructive pulmonary disease & $19(19.8)$ & $9(11.1)$ & 0.115 & $16(25.4)$ & $11(11.8)$ & 0.028 \\
\hline Deep-vein thrombosis & $41(42.7)$ & $28(34.6)$ & 0.269 & $25(39.7)$ & $36(38.7)$ & 0.903 \\
\hline Active cancer & $13(13.5)$ & $17(20.9)$ & 0.188 & $8(12.7 \%)$ & $19(20.4)$ & 0.210 \\
\hline Unprovoked PE & $65(67.7)$ & $35(43.2)$ & $<0.05$ & $44(69.8)$ & $46(49.5)$ & $<0.05$ \\
\hline Transient provoked PE & $18(18.8)$ & $29(35.8)$ & $<0.05$ & $11(17.5)$ & $28(30.1)$ & 0.073 \\
\hline PD at baseline & $9.6 \pm 4.2$ & $6.9 \pm 4.2$ & $<0.0001$ & $9.9 \pm 4.1$ & $6.9 \pm 4.3$ & $<0.0001$ \\
\hline 1-wk percent recovery (\%) & $30.0 \pm 30.7$ & $70.9 \pm 27.9$ & $<0.001$ & $28.9 \pm 27.4$ & $64.9 \pm 29.8$ & $<0.001$ \\
\hline $\mathrm{PA}-\mathrm{aO}_{2}$ at baseline $(\mathrm{mm} \mathrm{Hg})$ & $52.7 \pm 27.6$ & $49.4 \pm 37.9$ & 0.717 & $50.1 \pm 28.6$ & $49.9 \pm 37.5$ & 0.978 \\
\hline PAsP at baseline $(\mathrm{mm} \mathrm{Hg})$ & $47.7 \pm 10.1$ & $43.9 \pm 9.6$ & $<0.05$ & $48.8 \pm 10.4$ & $44.9 \pm 10.3$ & 0.05 \\
\hline
\end{tabular}

Univariate analysis: $P<0.05$ was considered significant. Qualitative data are expressed as numbers followed by percentages in parentheses; continuous data are expressed as mean \pm SD. 
TABLE 4

Variables Associated with Complete Recovery at 1 and 6 Months of Follow-up

\begin{tabular}{|c|c|c|c|c|}
\hline \multirow[b]{2}{*}{ Variable } & \multicolumn{2}{|c|}{$1 \mathrm{mo}$} & \multicolumn{2}{|c|}{$6 \mathrm{mo}$} \\
\hline & Odds ratio & $P$ & Odds ratio & $P$ \\
\hline PD baseline & $0.84(0.76-0.92)$ & $<0.0001$ & $0.80(0.72-0.89)$ & $<0.0001$ \\
\hline 1-wk percent recovery & $1.05(1.03-1.06)$ & $<0.0001$ & $1.04(1.02-1.05)$ & $<0.0001$ \\
\hline Unprovoked PE & $2.90(1.26-6.69)$ & $<0.05$ & - & - \\
\hline Age & - & - & $0.97(0.93-0.99)$ & 0.046 \\
\hline
\end{tabular}

PD recovery at the 1 -wk scan predicts a $3 \%$ increase in improvement probability; each 1 point of baseline PD score reduction predicts an $11 \%$ decrease in improvement probability. At variance with previous studies, older patients' age and underlying cardiac disease did not significantly influence the 6-mo perfusion recovery (26-28).

Perfusion impairment correlates with $\mathrm{PA}-\mathrm{aO}_{2}$ during follow-up. Intriguingly, measuring gas exchanges and calculating $\mathrm{PA}-\mathrm{aO}_{2}$ might be a good compromise in the event that pulmonary scintigraphy is not promptly available in the first days after diagnosis. Perfusion impairment also correlates with PAsP during the late phases of follow-up; PAsP is above the normal value in most patients at diagnosis and then tends to progressively decrease. Such a correlation is still present in a limited number of cases $1 \mathrm{y}$ after the index event; in fact, $10(5 \%)$ patients still had a pulmonary pressure over $40 \mathrm{~mm} \mathrm{Hg}$ and a residual arterial pulmonary obstruction. Of these, $6(3.3 \%)$ patients were still hypertensive $2 \mathrm{y}$ after PE and

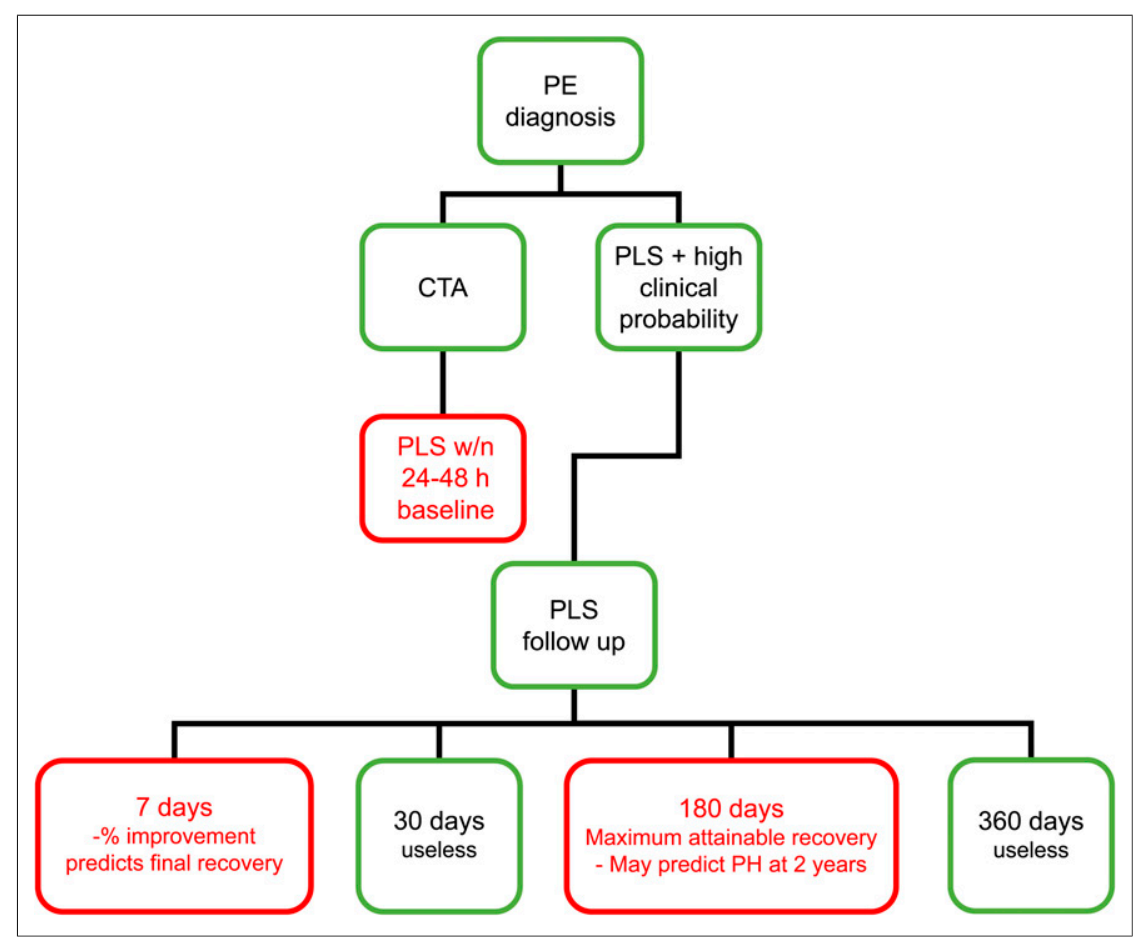

FIGURE 5. Suggested practical use of PLS in follow-up of acute PE. PH = pulmonary hypertension. Red boxes indicate suggested follow-up controls. were then considered to be affected by chronic thromboembolic pulmonary hypertension. Accordingly, we may speculate that patients who show incomplete recovery at 6 mo should undergo investigation for pulmonary hypertension and be followed up carefully to identify those who eventually will develop chronic thromboembolic pulmonary hypertension because a portion of them will develop this disease.

We also investigated a new parameter, the so-called hyperperfusion sign. This sign, previously described by our group (13), is due to the occurrence of abnormal areas of markedly increased perfusion in the upper and anterior lung regions because of a pulmonary blood flow shift from the lower and posterior lung regions presumably due to the predominant embolic obstruction in the lower lung regions. Hyperperfusion identifies regions with a low ventilation-perfusion ratio, in which perfusion is markedly increased and ventilation, though normal, is lower than perfusion. Under these conditions, perfusion is wasted in such areas (at a low ventilation-perfusion ratio), and this phenomenon may explain at least in part the arterial hypoxemia observed in acute PE (14). Hyperperfusion is possibly associated with acute pulmonary hypertension and tends to disappear after the first week of therapy. The detection of this finding after the acute phase of PE might be suggestive of PE recurrence. Furthermore, the presence of hyperperfusion 6 mo after acute PE correlates with persistently elevated PAsP; therefore, its presence in a follow-up scan suggests that surveillance should be set up for possible development of pulmonary hypertension in the following $18 \mathrm{mo}$.

One might argue that CTA may be more appropriate than PLS to follow patients with $\mathrm{PE}$ because it is more widely available and because almost all patients have the original diagnosis made by CTA. However, irradiation burden and poor correlation of such an examination with the evolution of pulmonary blood flow recovery make it less adapted to this purpose. PLS is less often used for diagnosis but has instead a great potential for following such patients, provided that PLS be systematically performed within 24-48 h after CTA diagnosis. In fact, Pesavento et al., after a disappointing experience with CT that showed the rate of residual 
obstruction to be much lower than expected and to not correlate with the endpoint (29), used PLS to successfully demonstrate the relation between residual vascular obstruction and the adverse clinical evolution (6).

The main limitations of this study are the relatively small sample size, the semiquantitative scoring system, and the singlecenter enrollment.

From a practical point of view, the ideal scintigraphic follow-up should consist of 3 essential steps (Fig. 5): the baseline study, since it indicates the severity of PE in the acute phase; the 1-wk follow-up, since it shows the early amount of reperfusion; and the 6-mo follow-up, since it shows the maximum attainable recovery of lung perfusion and can thus be considered the last control. As stated by Gottschalk in an editorial written on the Wartski and Collignon (26) article: "a chronic perfusion defect is a serious problem" and can complicate the management of patients with a previous diagnosis of PE and suspected recurrence in the absence of scintigraphic follow-up (30).

\section{CONCLUSION}

We believe this is the first systematic study that has investigated, with consecutive enrollment and a predefined time-control follow-up, pulmonary perfusion changes as evaluated on PLS in a large group of patients with a symptomatic episode of acute PE. PLS is a practical and useful way to follow such patients and might help with the decision to modify therapy in those at risk for incomplete restoration of pulmonary perfusion and for chronic thromboembolic pulmonary hypertension.

\section{DISCLOSURE}

No potential conflict of interest relevant to this article was reported.

\section{REFERENCES}

1. Heit JA, Mohr DN, Silverstein MD, Petterson TM, O'Fallon WM, Melton LJ III. Predictors of recurrence after deep vein thrombosis and pulmonary embolism: a population-based cohort study. Arch Intern Med. 2000;160:761-768.

2. Eichinger S, Weltermann A, Minar E, et al. Symptomatic pulmonary embolism and the risk of recurrent venous thromboembolism. Arch Intern Med. 2004; 164:92-96.

3. Huang W, Goldberg RJ, Anderson FA, Cohen AT, Spencer FA. Occurrence and predictors of recurrence after a first episode of acute venous thromboembolism: population-based Worcester venous thromboembolism study. J Thromb Thrombolysis. 2016;41:525-538.

4. Pengo V, Lensing AW, Prins MH, et al. Thromboembolic pulmonary hypertension study group. Incidence of chronic thromboembolic pulmonary hypertension after pulmonary embolism. $N$ Engl J Med. 2004;350:2257-2264.

5. Klok FA, van Kralingen KW, van Dijk AP, Heyning FH, Vliegen HW, Huisman MV. Prospective cardiopulmonary screening program to detect chronic thromboembolic pulmonary hypertension in patients after acute pulmonary embolism. Haematologica. 2010;95:970-975.

6. Pesavento R, Filippi L, Palla A, et al. The impact of residual pulmonary obstruction on the long-term outcome of patients with pulmonary embolism. Eur Respir J. 2017;49:1601980.

7. Miniati M, Pistolesi M, Marini C, et al. Value of perfusion lung scan in the diagnosis of pulmonary embolism: results of the prospective investigative study of acute pulmonary embolism diagnosis (PISA-PED). Am J Respir Crit Care Med. 1996;154:1387-1393.

8. Miniati M, Prediletto R, Formichi B, et al. Accuracy of clinical assessment in the diagnosis of pulmonary embolism. Am J Respir Crit Care Med. 1999;159:864-871.

9. Palla A, Ribas C, Rossi G, et al. The clinical course of pulmonary embolism patients anticoagulated for 1 year: results of a prospective, observational, cohort study. J Thromb Haemost. 2010;8:68-74.

10. Miniati M, Monti S, Bottai M, et al. Survival and restoration of pulmonary perfusion in a long-term follow-up of patients after acute pulmonary embolism. Medicine (Baltimore). 2006;85:253-262.

11. Sostman HD, Miniati M, Gottschalk A, Matta F, Stein PD, Pistolesi M. Sensitivity and specificity of perfusion scintigraphy combined with chest radiography for acute pulmonary embolism in PIOPED II. J Nucl Med. 2008;49:1741-1748.

12. Parker JA, Coleman RE, Grady E, et al. Society of Nuclear Medicine. SNM practice guideline for lung scintigraphy 4.0. J Nucl Med Technol. 2012;40:57-65.

13. Palla A, Marconi L, Bigazzi F, Pistolesi M. Lung scintigraphy in the diagnosis of pulmonary embolism: pathophysiologic and practical evidences. Clin Transl Imaging. 2014;2:363-367.

14. Santolicandro A, Prediletto R, Fornai E, et al. Mechanisms of hypoxemia and hypocapnia in pulmonary embolism. Am J Respir Crit Care Med. 1995;152:336347.

15. Hansson PO, Sorbo J, Eriksson H. Recurrent venous thromboembolism after deep vein thrombosis: incidence and risk factors. Arch Intern Med. 2000;160: 769-774.

16. Baglin $\mathrm{T}$, Luddington $\mathrm{R}$, Brown $\mathrm{K}$, Baglin $\mathrm{C}$. Incidence of recurrent venous thromboembolism in relation to clinical and thrombophilic risk factors: prospective cohort study. Lancet. 2003;362:523-526.

17. Klok FA, Mos IC, Huisman MV. Brain-type natriuretic peptide levels in the prediction of adverse outcome in patients with pulmonary embolism: a systematic review and meta analysis. Am J Respir Crit Care Med. 2008;178:425-430.

18. Lankeit M, Jimenez D, Kostrubiec M, et al. Validation of N-terminal pro-brain natriuretic peptide cut-off values for risk stratification of pulmonary embolism. Eur Respir J. 2014;43:1669-1677.

19. Becattini C, Vedovati MC, Agnelli G. Prognostic value of troponins in acute pulmonary embolism: a meta-analysis. Circulation. 2007;116:427-433.

20. Becattini C, Agnelli G, Vedovati MC, et al. Multidetector computed tomography for acute pulmonary embolism: diagnosis and risk stratification in a single test. Eur Heart J. 2011;32:1657-1663.

21. Trujillo-Santos J, den Exter PL, Go'mez V, et al. Computed tomographyassessed right ventricular dysfunction and risk stratification of patients with acute nonmassive pulmonary embolism: systematic review and meta-analysis. $J$ Thromb Haemost. 2013;11:1823-1832.

22. Kang DK, Thilo C, Schoepf UJ, et al. CT signs of right ventricular dysfunction: prognostic role in acute pulmonary embolism. JACC Cardiovasc Imaging. 2011; 4:841-849.

23. Quiroz R, Kucher N, Schoepf UJ, et al. Right ventricular enlargement on chest computed tomography: prognostic role in acute pulmonary embolism. Circulation. 2004;109:2401-2404.

24. Sanchez O, Trinquart L, Colombet I, et al. Prognostic value of right ventricular dysfunction in patients with haemodynamically stable pulmonary embolism: a systematic review. Eur Heart J. 2008;29:1569-1577.

25. Coutance G, Cauderlier E, Ehtisham J, Hamon M, Hamon M. The prognostic value of markers of right ventricular dysfunction in pulmonary embolism: a meta-analysis. Crit Care. 2011;15:R103.

26. Wartski M, Collignon M-A, for the THESEE Study Group. Incomplete recovery of lung perfusion after 3 months in patients with acute pulmonary embolism treated with antithrombotic agents. J Nucl Med. 2000;41:1043-1048.

27. Winebright JW, Gerdes AJ, Nelp WB. Restoration of blood flow after pulmonary embolism. Arch Intern Med. 1970;125:241-247.

28. Palla A, Pazzagli M, Manganelli D, et al. Resolution of pulmonary embolism: effect of therapy and putative age of emboli. Respiration. 1997;64:50-53.

29. Pesavento R, Visonà $\mathrm{A}$, Villalta $\mathrm{S}$, et al. Residual pulmonary obstruction and the risk of late complications in patients with pulmonary embolism. Thromb Res. 2016;137:228-230.

30. Gottschalk A. The chronic perfusion defect: our knowledge is still hazy, but the message is clear. J Nucl Med. 2000;41:1049-1050. 